\title{
Neural Correlates of Motion-induced Blindness in the Human Brain
}

\author{
Marieke L. Schölvinck and Geraint Rees
}

\begin{abstract}
Motion-induced blindness (MIB) is a visual phenomenon in which highly salient visual targets spontaneously disappear from visual awareness (and subsequently reappear) when superimposed on a moving background of distracters. Such fluctuations in awareness of the targets, although they remain physically present, provide an ideal paradigm to study the neural correlates of visual awareness. Existing behavioral data on MIB are consistent both with a role for structures early in visual processing and with involvement of high-level visual processes. To further investigate this issue, we used high field functional MRI to investigate signals in human low-level visual cortex and motion-sensitive area V5/MT while participants re-
\end{abstract}

\section{INTRODUCTION}

Motion-induced blindness (MIB) is a striking phenomenon in which a perceptually salient stationary visual target repeatedly disappears (and subsequently reappears) when superimposed on a field of moving distracters (Bonneh, Cooperman, \& Sagi, 2001). The repeated switches in awareness of the continuously present target render MIB an instance of bistable perception. Although this paradigm has been studied extensively in a number of behavioral studies, the neural mechanisms underlying this phenomenon have remained less well investigated.

One possibility is that MIB is similar to other types of perceptual fading and reflects neural activity in low-level visual cortex. For example, perceptual fading of stimuli presented on a static background will eventually occur with prolonged eccentric fixation (Troxler, 1804), which is thought to reflect adaptation very early in visual processing. However, the dynamics of MIB are rather different from Troxler fading. MIB can occur following very brief observation periods, disappearance of the target is rapid, and paradoxically, disappearance of the target occurs for longer when the contrast between target and distracters is greater (Bonneh et al., 2001). Behavioral results suggest that MIB is not likely to reflect local adaptation or lateral masking, as it persists for targets that are moving

University College London ported disappearance and reappearance of an MIB target. Surprisingly, perceptual invisibility of the target was coupled to an increase in activity in low-level visual cortex plus area V5/MT compared with when the target was visible. This increase was largest in retinotopic regions representing the target location. One possibility is that our findings result from an active process of completion of the field of distracters that acts locally in the visual cortex, coupled to a more global process that facilitates invisibility in general visual cortex. Our findings show that the earliest anatomical stages of human visual cortical processing are implicated in MIB, as with other forms of bistable perception. or flickering or when distracters and targets are spatially separated (Bonneh et al., 2001). Furthermore, when targets are invisible during MIB, they can still generate orientation specific aftereffects (Montaser-Kouhsari, Moradi, Zandvakili, \& Esteky, 2004) or negative afterimages (Hofstoetter, Koch, \& Kiper, 2006). This suggests that the locus of MIB lies beyond the cortical site at which these aftereffects are generated. Finally, MIB is sensitive to the cues that affect grouping (Hsu, Yeh, \& Kramer, 2004; Bonneh et al., 2001) and to the depth relations between target and distracters (Graf, Adams, \& Lages, 2002).

Taken together, these purely behavioral findings suggest that MIB does not reflect processes at the earliest stages of visual processing (e.g., primary visual cortex) but instead might originate in extrastriate or higher areas. Indeed, it has been proposed that MIB reflects competitive interactions between target and distracters that reflect their relative saliency (Bonneh et al., 2001). On such an account, MIB is one form of bistable perception and reflects the outcome of competition between neural mechanisms that signal incompatible percepts. This is an attractive proposal because it links MIB with other forms of bistable perception, such as binocular rivalry and generalized flash suppression, which appear phenomenally similar. However, it should be noted that an account of MIB based on relative salience of target and distracters is somewhat inconsistent with the observation that increasing target salience prolongs its invisibility (Bonneh et al., 
2001). Purely behavioral studies, therefore, do not provide a consistent prediction for the potential neural locus of MIB.

As a step toward investigating these issues, we used high field functional MRI to investigate activity changes in low-level visual cortex that were time locked to perceptual fluctuations in target visibility during MIB in human participants. Whole-brain analysis together with standard retinotopic mapping of visual cortex was used to characterize activity related to reported visibility of the target. To anticipate our findings, activity in low-level visual cortex (V1, V2), plus V5/MT, depended on the visibility of the target reported by our participants. Specifically, invisibility of the target was associated with increased activity in retinotopic regions of V1 and V2 representing the target plus a broader modulation of activity in $\mathrm{V} 2$ and area V5/MT. One possibility is that our findings reflect an active neural process of distracter-associated surface completion in MIB. More generally, they reinforce the notion that activity in low-level visual cortex is strongly associated with fluctuations in perceptual awareness (Mendola, Conner, Sharma, Bahekar, \& Lemieux, 2006; Lee, Blake, \& Heeger, 2005; Polonsky, Blake, Braun, \& Heeger, 2000), generalizing this notion to the phenomenon of MIB.

\section{METHODS}

\section{Observers and Stimuli}

Eight healthy volunteers with normal vision (20-30 years old; 6 women) gave written informed consent to participate in the experiment, which was approved by the local ethics committee. They viewed a yellow dot in their left upper visual field underneath a grid of blue distracter crosses rotating at $5.5 \mathrm{deg} / \mathrm{sec}$ round a central fixation dot (Figure 1A). This type of display configuration is known to invoke MIB (Caetta, Gorea, \& Bonneh, 2007; Graf et al., 2002; Bonneh et al., 2001). The grid (luminance $\left.8.81 \mathrm{~cd} / \mathrm{m}^{2}\right)$ was superimposed on a black background (luminance $0.10 \mathrm{~cd} / \mathrm{m}^{2}$ ). The yellow dot (luminance $11.23 \mathrm{~cd} / \mathrm{m}^{2}$ ) of size $0.8^{\circ}$ at $5.8^{\circ}$ eccentricity $\left(5^{\circ}\right.$ across and $3^{\circ}$ up) was flickering at $15 \mathrm{~Hz}$ to enhance its salience and to avoid adaptation aftereffects. The upper left visual field was chosen as MIB has been shown to be most robust at this location (Bonneh et al., 2001).

Observers were instructed to attend to the central fixation dot and to count the number of times it flashed red per run of scanning while simultaneously reporting the disappearance and the reappearance of the target by pressing one of two buttons with their right hand. The central fixation task was designed to reduce eye movements while not requiring further button presses. Furthermore, we reasoned that this dual task situation (MIB task and central fixation task) might direct attention away from the MIB target and might, therefore, minimize any potential fluctuations in attention associated with perception of the target.
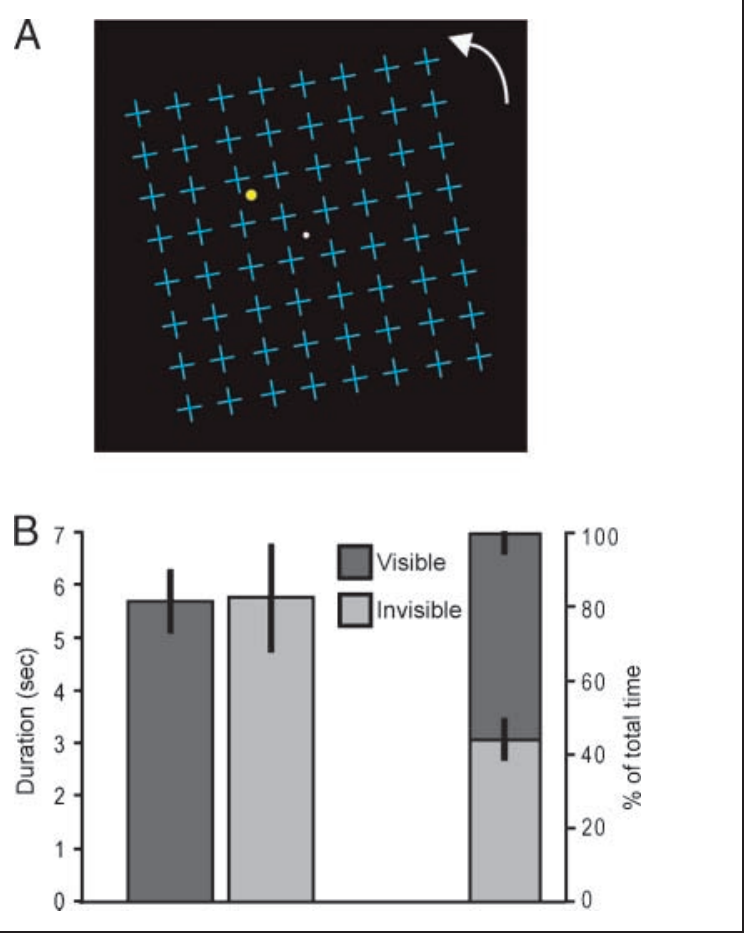

Figure 1. Stimulus configuration and behavioral data. (A) Participants fixated centrally (white dot) and viewed a yellow flickering dot placed in the left upper quadrant and surrounded by blue crosses. In the dynamic display, the entire field of cross distracters rotated clockwise or counterclockwise about the fixation point. Participants were required to indicate by button presses when the yellow flickering dot became visible or invisible to them. (B) Durations and percent total time of visibility and invisibility of the target are shown for the scanning experiment, averaged across participants. Error bars represent the SEM.

\section{Procedure}

Participants were tested extensively before the scanning experiment to ensure they could experience MIB and assign consistent responses to the different perceptual states. They performed a further practice block in the scanner before data recording. Subsequently, they completed nine 6-min runs of scanning. A run started with $10 \mathrm{sec}$ when only the field of rotating blue crosses was presented (target absent), after which the yellow flashing dot appeared. Participants then indicated continuously by button presses when the target either became visible (target visible) or became invisible (target invisible). In addition, during periods while the participant had indicated that the target was visible, the target would be physically removed from the display for periods of $10 \mathrm{sec}$ five more times during the scanning run at random intervals. These extra absent periods were included to determine that participants were correctly following instructions; upon subsequent debriefing, all participants declared that they had not realized that the target was sometimes not only perceptually but also physically absent. Theoretically, during these absent periods, subjects could perceive the target as being visible (fourth condi- 
tion); however, analysis of their button presses showed that this was never the case. The button presses made by participants were thus used to divide the viewing epochs into the remaining three conditions (target visible, target invisible, and target absent). As the target was flickering, there were no target-contingent aftereffects. Halfway through each run, the rotation direction of the distracter field was reversed to prevent a strong aftereffect from the rotating crosses.

Every three runs of task were followed by a run of ROI localizer (see below). Standard retinotopic maps (see below) and T1-weighted structural scans were acquired in a separate session.

\section{Retinotopic Mapping and ROI Localizer}

Each participant was scanned using a conventional retinotopic mapping procedure, viewing a contrast-reversing checkerboard stimulus presented as horizontal or vertical wedges placed to cover either horizontal or vertical meridians in successive scanning blocks (Wandell, Chial, \& Backus, 2000; Teo, Sapiro, \& Wandell, 1997; Sereno et al., 1995) for V1 and V2 localization. V5/MT was localized using expanding and contracting rings of dots (Rees, Frith, \& Lavie, 1997). Finally, the retinotopic location of the target representation in low-level visual areas was determined by measuring brain activity while participants viewed a white dot on a black background (luminance $=13.64$ and $0.10 \mathrm{~cd} / \mathrm{m}^{2}$ ) flickering at $15 \mathrm{~Hz}$, of identical size and location as the dot used in the actual experiment. This was shown for three 5-min runs, each consisting of alternating 15 -sec periods of the flickering dot and a blank screen. To ensure stable fixation, we required participants to press a button as soon as a small red dot flashed on either side of a fixation cross throughout these runs.

\section{fMRI Data Acquisition and Analysis}

A 3-T Allegra MRI scanner (Siemens Medical Systems, Erlangen, Germany) with a standard transmit-receive head coil was used to acquire functional data with a single-shot gradient-echo isotropic high-resolution EPI sequence (matrix size $=128 \times 128$; field of view $=192 \mathrm{~mm}$; in-plane resolution $=1.5 \mathrm{~mm}$; 32 slices with interleaved acquisition; slice thickness $=3 \mathrm{~mm}$; echo time $=30 \mathrm{msec}$; acquisition time per slice $=102 \mathrm{msec}$; repetition time $=$ $2040 \mathrm{msec}$ ). During scanning, eye position was continually sampled at $60 \mathrm{~Hz}$ using an ASL 504 LRO infrared videobased MRI compatible eye tracker (Applied Science Laboratory, Bedford, MA).

Functional data were analyzed using SPM5 (http:// www.fil.ion.ucl.ac.uk/spm/software/spm5/). The first five images of each series were discarded from further analyses to allow for T1 equilibration effects. Preprocessing of the data involved realignment of each scan to the first scan of the first experimental run and coregistration of the functional data to the structural scan. Data were normalized to an EPI template in MNI stereotactic space and smoothed by a 6-mm kernel for whole-brain analysis; the ROI localizer and individual retinotopic and V5/MT analyses were carried out on nonnormalized, unsmoothed data. Data were not smoothed because of the small size of the ROI localizer regions. The data were filtered with a 128-sec cutoff, high-pass filter to remove low-frequency noise and adjusted for global changes in activity. The timing of button presses was used on a per-participant basis to construct three participant-specific regressors that represented hypothesized brain activity associated with each of the three behaviorally defined conditions (target visible, target invisible, and target absent). In an additional analysis, subjects' individual RTs were calculated from the time of their button presses to the "target absent" condition; these RTs were then subtracted from the time of all button presses to arrive at a better estimate of the "real" time of the perceptual switches. These corrected times were then used as regressors in the analysis. In a second additional analysis, variance associated with eye blinks was modeled and removed as an extra regressor of no interest. Movement parameters in the three directions of motion and three degrees of rotation were included as confounds. These regressors were convolved with a synthetic hemodynamic response function and entered into a general linear model. Parameter values for each regressor were estimated for each participant independently. In the group analysis, these estimates were entered treating participants as a random factor, using a one-sample $t$ test across participants.

For two participants, eye data could not be analyzed due to technical difficulties. Eye movements were defined as variance round the mean $x$ and $y$ position of the eye and eye blinks as an absence of signal. Statistical analysis was done to examine differences in eye movements and blinks between the various experimental conditions.

\section{Retinotopic Analysis}

Retinotopic cortical areas were identified using mrGray (Wandell et al., 2000; Teo et al., 1997). This yielded maps of functionally defined visual areas V1, V2, and V3 for each participant. These maps were combined with activation images of the ROI localizer to reveal retinotopic regions in V1 and V2 representing the spatial location of the target. Retinotopic regions in V3 corresponding to the target location could not be identified in all subjects because the target was small and eccentrically placed, making it more difficult to localize in higher visual areas (Dougherty et al., 2003). This process thus yielded ROIs representing the spatial location of the target in V1 and V2 for each participant. We then used these ROIs to extract estimates of activity in each of the three experimental conditions of interest (target visible, target invisible, and target absent) obtained from the multiple linear regression analysis described above. Activity estimates were averaged across 
the voxels within the ROI and subjected to repeated measures ANOVAs and subsequent planned paired $t$ tests to test for differences between conditions.

To compare activation in these ROI localizer regions with activation in regions of comparable size and eccentricity that did not represent the spatial location of the target, we sampled regions of the same size (spatial extent) at a corresponding eccentricity (based on visual inspection of the retinotopic maps) from the left dorsal V1 and V2 (representing the lower right visual quadrant, diagonally opposite the visual field location of the target) for each participant individually. Activity associated with each of the three experimental conditions in these control ROIs was assessed in a similar fashion as for the target ROI. We further examined additional, larger control regions in which activity was extracted and averaged across the whole of right and left V1 and V2 (i.e., regions contralateral and ipsilateral to the visual field location of the MIB target).

The location of V5/MT was determined for each participant by subtracting activation during the static dots from activation during the expanding and contracting dots (Rees et al., 1997), taking the peak voxel in the regions closest to where MT has commonly been reported (Morrone et al., 2000) and taking all gray matter voxels in a sphere of $8 \mathrm{~mm}$ round these peak voxels. These spheres were used as masks for all voxels activated by our stimulus (distracters and target; $F$ contrast of all our conditions versus the rest period) to reveal voxels in right and left V5/MT that were activated in our experiment.

\section{RESULTS}

\section{Behavioral Data}

On average across all scanning runs, periods where the target was invisible to participants due to MIB lasted for a mean duration of $5.81 \mathrm{sec}(S E=0.98 \mathrm{sec})$ and those where the target was visible for a mean duration of $5.74 \mathrm{sec}(S E=$ $0.55 \mathrm{sec}$; Figure 1B; individual participant data are shown in Supplementary Table 1). In total, the target was invisible due to MIB (excluding target absent periods) for $44.3 \%$ ( $S E=5.0 \%$ ) of the time (Figure 1B). For each participant, we estimated their RT to a perceptual disappearance or appearance due to MIB by measuring their RT to respond to actual physical removal of the target. Their average RT was $1.67 \mathrm{sec}(S E=0.17 \mathrm{sec})$; the flicker and peripheral location of the target, a conservative response strategy, and an engagement of participants in the simultaneous central fixation task are all consistent with this relatively long RT.

\section{Neural Activity Associated with MIB in Retinotopic Target Locations}

We investigated whether there were any differences in activation in the localizer ROIs (Figure 2A) comparing the experimental conditions (target visible, target invisible, and target absent). Repeated measures ANOVAs (GreenhouseGeisser corrected) on measures of the BOLD activity within these ROIs (Figure 2B) showed significant differences between the three experimental conditions in both V1 and V2 ROI locations, $F(2,14)=22.121, p=.001$ and $F(2,14)=$ $11.659, p=.008$ for V1 and V2, respectively. Planned $t$ tests revealed that these differences reflected a significant increase in activity when the target was physically present and reported as visible compared with when it was physically absent (and reported as "invisible"), $t(7)=4.583$, $p=.003$ for V1 and $t(7)=3.923, p=.006$ for V2 (Figure $2 \mathrm{~B})$. More importantly, activity was also significantly elevated when the target was physically present but invisible due to MIB compared with the visible condition, $t(7)=3.804, p=.007$ for $\mathrm{V} 1$ and $t(7)=2.654, p=.033$ for V2 (Figure 2B). Activity in this invisible condition was also increased compared with the absent condition, $t(7)=$ $5.125, p=.001$ for V1 and $t(7)=3.523, p=.010$ for V2 (Figure $2 \mathrm{~B}$ ). Such a pattern was highly consistent across our participants, being observed in seven of eight participants. Qualitatively, visual inspection of time courses (Figure 2C) from the three conditions time locked to reports of perceptual transitions show that these significant differences were associated with a large initial decrease and a slightly smaller initial increase in the absent and invisible condition, respectively.

Both the V1 and the V2 localizer ROIs showed a similar pattern of activity (Figure 2B) associated with the experimental conditions, and this pattern persisted in a subsequent control analysis when the timing of the three experimental conditions defined by button presses were corrected for the individual RTs of each participant assessed from physical disappearances of the target (see Methods section and Supplementary Figure 1).

\section{Activity in Other Regions of Visual Cortex}

To determine whether these activity differences were retinotopically specific or whether they reflected more general activity differences in visual cortex, we investigated BOLD activity in a number of control regions: the (similarly sized) control ROIs in V1 and V2 ipsilateral to the stimulus, the whole of ipsilateral V1 and V2 activated by the stimulus (target and distracters), and the right and left V5/MT.

Activity in the control ROIs in V1 and V2 (Figure 2D) qualitatively showed a similar pattern, but of much lower amplitude, as for the localizer regions. However, these qualitative differences between conditions were not significant in V1, $F(2,14)=.681, p=.497$, although they trended toward significance in V2, $F(2,14)=3.541, p=.078$ (Figure 2E). Post hoc planned $t$ tests also showed no significant differences between any of the conditions, all $t(7)<1.886$, all $p>.101$, apart from the difference between the visible and the invisible condition in $\mathrm{V} 2, t(7)=2.462, p=.043$. Time courses for the three conditions in these control ROIs were qualitatively very similar (Figure 2F). 
A

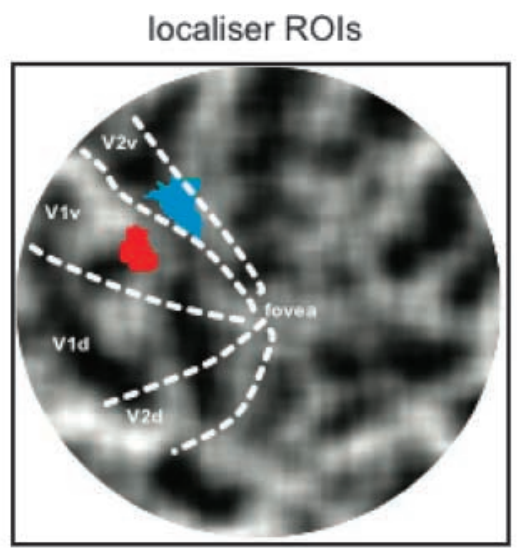

B
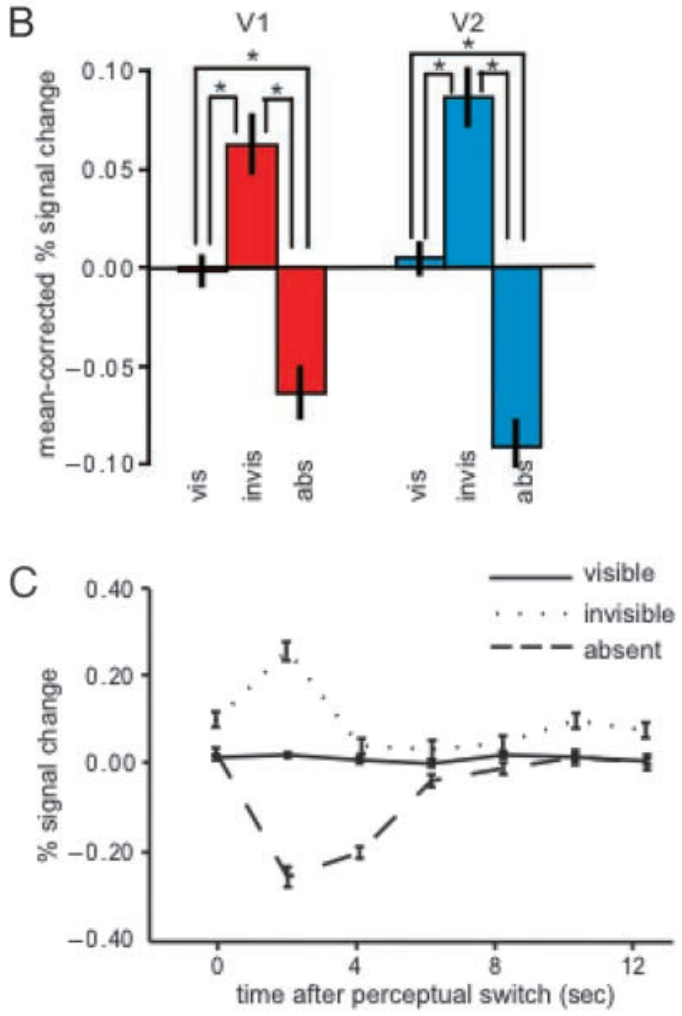

D

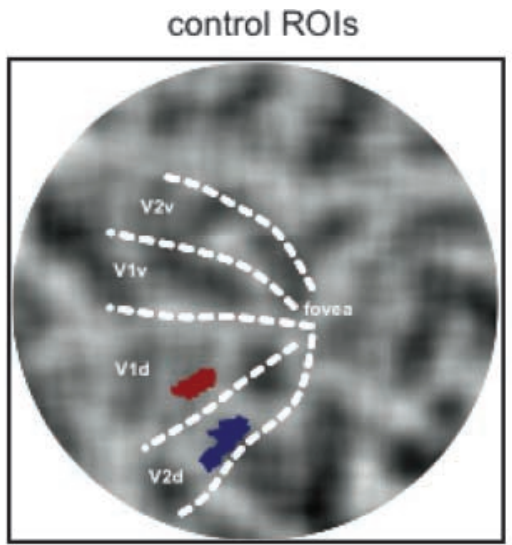

E

V1

V2
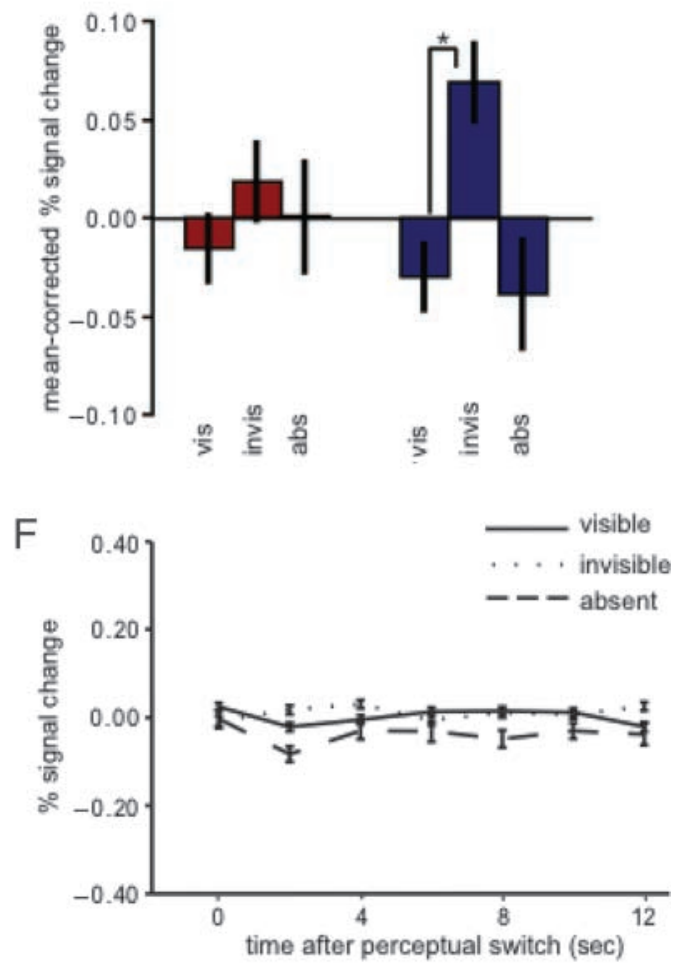

Figure 2. BOLD signal in localizer and control ROIs. The retinotopic regions in visual cortex (localizer ROIs) representing the spatial location of the target for a representative participant are shown on a flattened representation of the right (contralateral) visual cortex (A; V1 is bright red, V2 is bright blue). Mean percent BOLD signal change (mean corrected and compared with resting baseline) in these regions for the three conditions [vis (target visible)-invis (target invisible)-abs (target absent)] are plotted in B; bright red bars represent the V1 localizer, and bright blue bars represent the V2 localizer. Error bars represent the group SEM. Raw data are shown in Supplementary Figure 2. Time courses of these three conditions are shown in C for the localizer region in V1. Time zero signals the time of the perceptual switch; time courses for the visible, invisible, and absent condition are shown in straight, dotted, and dashed lines, respectively. Retinotopic regions of the same size but representing the right lower visual quadrant (control ROIs) are shown in D, on a flattened representation of the left visual cortex for the same representative participant (V1 is dark red, V2 is dark blue). Mean percent BOLD signal change (mean corrected and compared with resting baseline) for the three conditions (target visible-invisible-absent) is plotted in E; dark red bars represent the V1 control ROI, and dark blue bars represent the V2 control ROI. Again, raw data are shown in Supplementary Figure 2. Time courses of the three conditions for the control ROI in V1 are shown in F; conventions are the same as in C.

As some weak and nonsignificant changes in activity associated with experimental conditions were present in these control ROIs, we explicitly tested whether the activity differences we observed in localizer ROIs were significantly different from control ROIs. Critically, the interaction between condition (visible, invisible, absent) and region (localizer, control) was significant in V1 and trended toward significance in V2, $F(2,14)=9.510, p=$ .003 and $F(2,14)=3.582, p=.087$ for V1 and V2, respectively. Hence, MIB-associated modulation of activity in 
retinotopic regions representing the target was significantly greater than in control ROIs in V1 but not in V2.

Activity in the whole of ipsilateral V1 and V2 showed a similar pattern of activity that was nonsignificant in V1 and almost significant in V2, $F(2,14)=2.584, p=.136$ and $F(2,14)=4.515, p=.052$ for $\mathrm{V} 1$ and $\mathrm{V} 2$, respectively (see Figure 3A). However, post hoc planned $t$ tests showed a significant difference between the visible and the invisible condition in both regions, $t(7)=2.761, p=.028$ and $t(7)=3.378, p=.012$ for $\mathrm{V} 1$ and $\mathrm{V} 2$, respectively (see Figure 3). Activity in right V5/MT showed a similar pattern to the ROI localizer regions in V1 and V2. There was a significant difference between the three experimental conditions, $F(2,14)=10.791, p=.010$, which was due to greater activity in the target invisible condition compared with the target visible condition, $t(7)=6.085, p<.001$, or compared with the target absent condition, $t(7)=4.677$, $p=.002$ (Figure 3B). There were no significant differences between the target visible and the target absent condition where (in both conditions) the distracters were always present, $t(7)=0.924, p=.386$. Activity in left V5/MT showed no significant difference between any of the conditions, $F(2,14)=2.476, p=.128$ (Figure $3 \mathrm{~B}$ ).

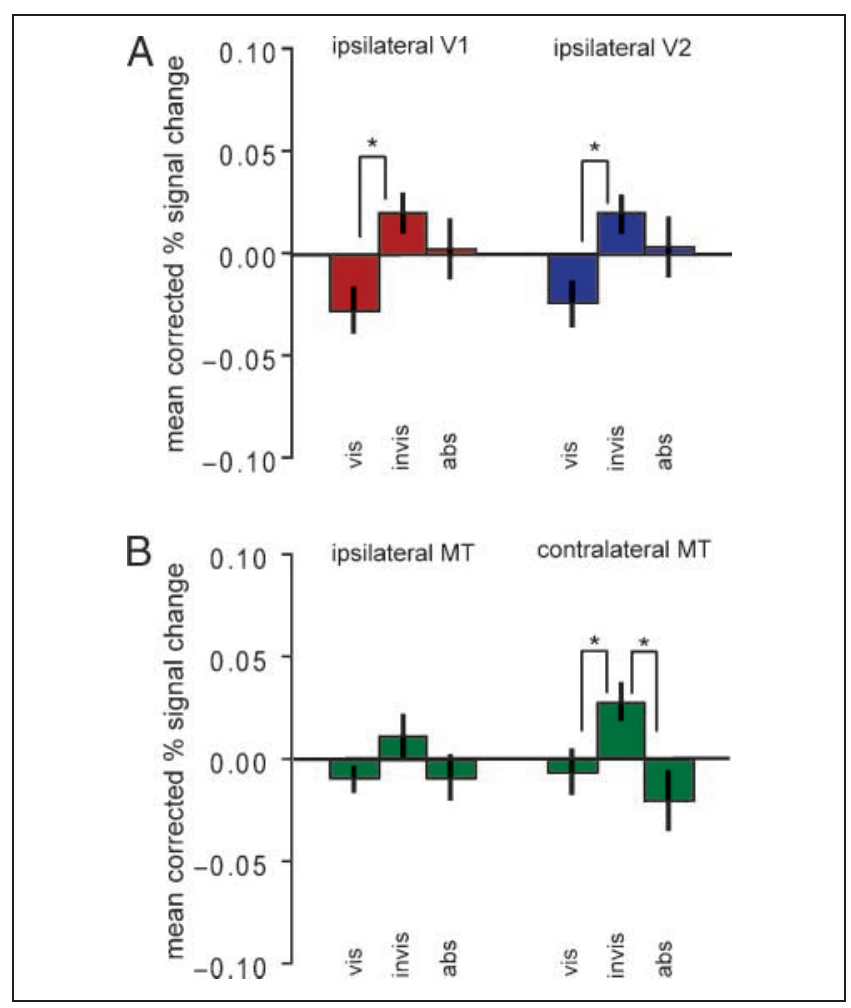

Figure 3. BOLD signal in other control regions. Mean percent BOLD signal change (mean corrected and compared with resting baseline) is shown for the three conditions [vis (target visible)-invis (target invisible)-abs (target absent)] for the whole of ipsilateral (left) V1 and V2 (A) and for ipsilateral (left) and contralateral (right) V5/MT (B). Error bars represent the group SEM. Raw data are shown in Supplementary Figure 2

\section{Whole-brain Analysis}

For completeness, we also performed a whole-brain analysis to identify any areas outside low-level visual cortex that might show modulation by perceptual state during MIB. It should be noted that such an analysis necessarily has lower sensitivity due to the much larger number of multiple comparisons and absence of a prior hypothesis. Outside occipital cortex (see retinotopic analyses above) and at a statistical threshold of $p<.05$ (FDR corrected for multiple comparisons across the whole brain), neither the comparison of the invisible versus visible condition nor the comparison of the visible versus absent condition yielded any suprathreshold clusters of voxels. When the threshold was dropped to $p<.0001$ uncorrected, the location in V1 corresponding to the target was significantly activated in all expected comparisons (invisible $>$ visible, invisible $>$ absent, visible $>$ absent), MNI coordinates [12 -78 -3], 13 voxels, $Z>4.97, p<.0001$. These comparisons also showed significant activation in right and left parietal cortex at this threshold (MNI coordinates [30 - 4254 ] and $[-30-4254], \sim 15$ voxels, $Z>5.52, p<.0001)$. When looking specifically at perceptual switches, the left frontal cortex was activated (MNI coordinates [ $-15-666]$, 15 voxels, $Z=5.33, p<.0001)$. However, as these areas were not hypothesized a priori to be involved in MIB and the activations were obtained at quite a liberal threshold, these results will not be discussed any further.

\section{Fixation and Eye Blink Data}

Repeated measures ANOVAs of eye position and average number of eye blinks per second revealed that there were no significant differences between the three conditions, $F(2,10)=1.916, p=.222$ and $F(2,10)=.352$, $p=.675$, respectively. In addition, modeling eye blinks as an additional regressor of no interest did not qualitatively alter our findings (data not shown). Hence, the differences in activity we observed in low-level visual cortex cannot be explained by differences in retinal input caused by eye movements or blinks at the resolution of our eye tracker.

\section{DISCUSSION}

In the present study, we have shown that disappearance of a target in MIB was associated with an increase in activity in low-level visual cortex, which was largest in retinotopic regions of $\mathrm{V} 1$ and $\mathrm{V} 2$ corresponding to the cortical representation of the target, plus in V5/MT contralateral to the target. These activity increases were specific to the retinotopic location of the target representation in $\mathrm{V} 1$, but in V2 a broader pattern of activity increases associated with invisibility was seen that also affected control ROIs distant from the target representation. In contrast, activity in retinotopic regions corresponding to the 
target location in V1 and V2 was reduced when the target was physically removed from the display. The striking differences in activity associated with the perceptually comparable situations of the target being invisible through MIB and through physical removal suggest that the neural processes underlying perceptual disappearances may reflect not merely target absence but also an additional mechanism that is specific to this state of target invisibility.

It has been suggested that MIB might reflect the disruption of attentional switching mechanisms, thus implying an interaction between higher and lower cortical areas (Bonneh et al., 2001). Others have argued that MIB results from surface completion associated with the moving distracters (Graf et al., 2002), which implies neural competition between representations of the moving distracters and the target (Libedinsky, Savage, \& Livingstone, 2009; Keysers \& Perrett, 2002). The small size of the target in our study and the inherently limited spatial resolution of fMRI make it difficult to disentangle distracter from target activation in our localizer regions. We cannot, therefore, isolate the separate contributions of target- and distracterrelated activity in our results. As such, the initial increase in activity when the target disappears (Figure 2C) might be related to the specific position or motion of the distracter grid. However, this seems less likely given that the grid was uniform and rotating at a constant speed. One way to distinguish between target- and distracter-related activity in a future experiment would be frequency-tagged MEG, which has been used successfully to distinguish between target- and background-related activity in perceptual completion (Weil, Kilner, Haynes, \& Rees, 2007). A possible mechanism that could account for the enhanced activations correlated with invisibility that we observe is neural competition between target and distracters (Libedinsky et al., 2009; Keysers \& Perrett, 2002); the activations could reflect an active process of completion of the perceived field of distracters into a perceptual surface occluding the target (Graf et al., 2002). There is physiological evidence for surface representation early in visual processing; V1 neurons show responses to the presence of contextual lines parallel and in proximity to a line within their receptive field (Kapadia, Ito, Gilbert, \& Westheimer, 1995), and V2 neurons use local depth information to integrate contours and segment the visual input into surfaces (Bakin, Nakayama, \& Gilbert, 2000). Interestingly, V1 and V2 neurons also show increased activity when an illusory contour is perceived in their receptive field (Lee \& Nguyen, 2001), consistent with an active contourcompletion mechanism giving rise to an increase in population activity. Thus, the increases in activity that we observe might result from local completion of the distracter field in the target region.

Strikingly, perceptual invisibility during MIB was also associated with modulation of activity in ipsilateral control ROIs, consistent with earlier findings (Donner, Sagi, Bonneh, \& Heeger, 2008; Hsieh, Caplovitz, \& Tse, 2005). Although statistically not significant in V1, in V2 the changes were more pronounced and reached statistical significance. Importantly, this modulation was observed in regions of visual cortex that do not represent the same visual field as where the MIB target was shown. Consistent with this, these regions showed only significant differences in activity when the visibility of the target changed and no significant difference between the physical presence versus absence of the target. Also, the invisible and the absent condition required the same response behavior. This indicates that these ipsilateral control regions do not respond to the physical presence of the target or to the detection of its disappearances but only to changes in visibility associated with MIB, which are therefore associated to some extent with a more generalized nonretinotopic modulation of activity. This is reminiscent of the global modulation of activity observed in low-level visual areas in a similar study investigating MIB (Donner et al., 2008), although our pattern of activity is opposite in sign. One possibility is that our increases in activity reflect a general arousal associated with perceptual invisibility of the target. However, this cannot account for why the changes are most prominent in V2 nor why similar elevation in activity for invisible versus visible is not also seen for the perceptually comparable situations of target absent versus visible. Another possibility is the differences in distracters used; the distracters in the present study formed a structured field of similarly oriented lines organized into crosses, whereas Donner et al. (2008) used an unstructured cloud of dots. These differences might result in differences in neural processing, resulting in qualitatively different results.

Interesting in this respect is the fact that although behavioral evidence suggests that MIB and perceptual completion associated with artificial scotomas are susceptible to similar factors (Hsu, Yeh, \& Kramer, 2006), the retinotopic increase in population-level neural activity we found associated with MIB contrasts with reports of decreases in activity associated with perceptual completion of an artificial scotoma (Weil, Watkins, \& Rees, 2008; Weil et al., 2007; Mendola et al., 2006). The resolution of functional MRI in humans is relatively low; so one possibility is that differences in population activity indexed by the BOLD signal reflect a combination of signals from different neural processes. For example, in perceptual completion associated with an artificial scotoma, it is hypothesized that a more rapid process of surface completion may follow a slow process of neural adaptation at the border between a target and its surround. Functional MRI, even at high spatial resolution, would be expected to conflate the two signals from our relatively small target, and so differences between MIB and other paradigms might not reflect different underlying mechanisms (e.g., surface completion) but instead a different balance or combination of individual neuronal properties underlying the phenomena. Future work will need to carefully manipulate stimulus properties to attempt to dissect out potential components of the underlying neural mechanisms. 
The present study failed to find strong evidence for the involvement of higher level areas in MIB. As noted above, this might be due to the conservative analysis strategy used or the small size of the target. If higher level areas were involved, though, they might influence the activity changes in low-level visual cortex through a feedback mechanism. Perceptual switches in bistable perception paradigms have been suggested to rely at least partly on feedback from higher level areas to low-level visual cortex (Windmann, Wehrmann, Calabrese, \& Güntürkün, 2006; Blake \& Logothetis, 2002; Lumer, Friston, \& Rees, 1998). Indeed, a possible role of feedback in MIB seems to be supported by the modulation of activity in visual areas $\mathrm{V} 4$ and the intraparietal sulcus by subjective target disappearances (Donner et al., 2008). Alternatively, the activity changes in low-level visual cortex that we observe might be the result of long-range horizontal connections within V1 that play a role in contour integration (Stettler, Das, Bennett, \& Gilbert, 2002); that is, they might result from local processing in low-level visual cortex. This notion must be treated with caution, however, because several electrophysiological studies have revealed an apparent discrepancy between the BOLD signal and the local neuronal activity in V1 during perceptual suppression (Maier et al., 2008; Wilke, Logothetis, \& Leopold, 2006).

One key process that can exert influences on activity in low-level visual cortex through feedback is spatial attention (Brefczynski \& DeYoe, 1999; Ghandi, Heeger, \& Boynton, 1999; Somers, Dale, Seiffert, \& Tootell, 1999). However, voluntary direction of spatial attention cannot easily account for our findings of increased activity in retinotopic regions representing the target associated with invisibility during MIB. First of all, participants were continuously involved in dividing their attention between a demanding task at fixation and reporting the MIB target. Moreover, there were also strong differences in activity between the perceptually comparable (and so equally likely to lead to voluntary shifts of attention) situations of the target being invisible due to MIB versus the target being invisible due to physical removal. However, it is important to note that although these findings indicate that voluntary attention may not play a role in MIB per se, they do not rule out the fact that attention can influence the timing of the perceptual disappearances and reappearances, as we have recently shown (Schölvinck \& Rees, 2009).

In conclusion, we present evidence for an involvement of low-level visual areas in MIB. We show that perceptual, but not physical, disappearance of the target is coupled to an increase in activity in retinotopic locations in V1 and V2 representing the target plus motion area V5/MT contralateral to the target. We hypothesize that these findings are consistent with a local active process of completion of the field of distracters underlying MIB but also point out the more general involvement of large regions of visual cortex; MIB thus seems to be implicated both at a local and at a global level.

\section{Acknowledgments}

This work was supported by the Wellcome Trust. The authors thank E. Anderson and B. Bahrami for advice and comments on the article.

Reprint requests should be sent to Marieke Schölvinck, Institute of Cognitive Neuroscience, 17 Queen Square, London WC1N 3AR, England, or via e-mail: M.Scholvinck@ucl.ac.uk.

\section{REFERENCES}

Bakin, J. S., Nakayama, K., \& Gilbert, C. D. (2000). Visual responses in monkey areas V1 and V2 to three-dimensional surface configurations. Journal of Neuroscience, 20, 8188-8198.

Blake, R., \& Logothetis, N. K. (2002). Visual competition. Nature Reviews Neuroscience, 3, 13-21.

Bonneh, Y. S., Cooperman, A., \& Sagi, D. (2001). Motion-induced blindness in normal observers. Nature, 411, 798-801.

Brefczynski, J. A., \& DeYoe, E. A. (1999). A physiological correlate of the "spotlight" of visual attention. Nature Neuroscience, 2, 370-374.

Caetta, F., Gorea, A., \& Bonneh, Y. (2007). Sensory and decisional factors in motion-induced blindness. Journal of Vision, 7, 1-12.

Donner, T. H., Sagi, D., Bonneh, Y. S., \& Heeger, D. J. (2008). Opposite neural signatures of motion-induced blindness in human dorsal and ventral visual cortex. Journal of Neuroscience, 28, 10298-10310.

Dougherty, R. F., Koch, V. M., Brewer, A. A., Fischer, B., Modersitzki, J., \& Wandell, B. A. (2003). Visual field representations and locations of visual areas V1/2/3 in human visual cortex. Journal of Vision, 3, 586-598.

Ghandi, S. P., Heeger, D. J., \& Boynton, G. M. (1999). Spatial attention affects brain activity in human primary visual cortex. Proceedings of the National Academy of Sciences, U.S.A., 96, 3314-3319.

Graf, E. W., Adams, W. J., \& Lages, M. (2002). Modulating motion-induced blindness with depth-ordering and surface completion. Vision Research, 42, 2731-2735.

Hofstoetter, C., Koch, C., \& Kiper, D. C. (2006). Motion induced blindness does not affect the formation of negative afterimages. Consciousness and Cognition, 13, 691-708.

Hsieh, P., Caplovitz, G. P., \& Tse, P. U. (2005). Neuronal activity varies with motion-induced blindness in ipsilateral and contralateral retinotopic cortex and contralateral HMT+ [Abstract]. Society for Neuroscience Abstracts, 136.6.

Hsu, L. C., Yeh, S. L., \& Kramer, P. (2004). Linking motion-induced blindness to perceptual filling-in. Vision Research, 44, 2857-2866.

Hsu, L. C., Yeh, S. L., \& Kramer, P. (2006). A common mechanism for perceptual filling-in and motion-induced blindness. Vision Research, 46, 1973-1981.

Kapadia, M. K., Ito, M., Gilbert, C. D., \& Westheimer, G. (1995). Improvement in visual sensitivity by changes in local context: Parallel studies in human observers and in V1 of alert monkeys. Neuron, 15, 843-856.

Keysers, C., \& Perrett, D. I. (2002). Visual masking and RSVP reveal neural competition. Trends in Cognitive Sciences, 6, 120-125.

Lee, S. H., Blake, R., \& Heeger, D. J. (2005). Traveling waves of activity in primary visual cortex during binocular rivalry. Nature Neuroscience, 8, 22-23.

Lee, T. S., \& Nguyen, M. (2001). Dynamics of subjective contour formation in the early visual cortex. Proceedings of the National Academy of Sciences, U.S.A., 98, 1907-1911. 
Libedinsky, C., Savage, T., \& Livingstone, M. (2009). Perceptual and physiological evidence for a role for early visual areas in motion-induced blindness. Journal of Vision, 9, 1-10.

Lumer, E. D., Friston, K. J., \& Rees, G. (1998). Neural correlates of perceptual rivalry in the human brain. Science, 280, $1930-1934$

Maier, A., Wilke, M., Aura, C., Zhu, C., Ye, F. Q., \& Leopold, D. A. (2008). Divergence of fMRI and neural signals in V1 during perceptual suppression in the awake monkey. Nature Neuroscience, 11, 1193-1200.

Mendola, J. D., Conner, I. P., Sharma, S., Bahekar, A., \& Lemieux, S. (2006). fMRI measures of perceptual filling-in in the human visual cortex. Journal of Cognitive Neuroscience, 18, 363-375.

Montaser-Kouhsari, L., Moradi, F., Zandvakili, A., \& Esteky, H. (2004). Orientation-selective adaptation during motion-induced blindness. Perception, 33, 249-254.

Morrone, M. C., Tosetti, M., Montanaro, D., Fiorentini, A., Cioni, G., \& Burr, D. C. (2000). A cortical area that responds specifically to optic flow, revealed by fMRI. Nature Neuroscience, 3, 1322-1328.

Polonsky, A., Blake, R., Braun, J., \& Heeger, D. J. (2000). Neuronal activity in human primary visual cortex correlates with perception during binocular rivalry. Nature Neuroscience, 3, 1153-1159.

Rees, G., Frith, C. D., \& Lavie, N. (1997). Modulating irrelevant motion perception by varying attentional load in an unrelated task. Science, 278, 1616-1619.

Schölvinck, M. L., \& Rees, G. (2009). Attentional influences on the dynamics of motion-induced blindness. Journal of Vision, 9, 1-9.

Sereno, M. I., Dale, A. M., Reppas, J. B., Kwong, K. K. Belliveau, J. W., Brady, T. J., et al. (1995). Borders of multiple visual areas in humans revealed by functional magnetic resonance imaging. Science, 268, 889-893.
Somers, D. C., Dale, A. M., Seiffert, A. E., \& Tootell, R. B. H. (1999). Functional MRI reveals spatially specific attentional modulation in human primary visual cortex. Proceedings of the National Academy of Sciences, U.S.A., 96, 1663-1668.

Stettler, D. D., Das, A., Bennett, J., \& Gilbert, C. D. (2002). Lateral connectivity and contextual interactions in macaque primary visual cortex. Neuron, 36, 739-750.

Teo, P. C., Sapiro, G., \& Wandell, B. A. (1997). Creating connected representations of cortical gray matter for functional MRI visualization. IEEE Transactions on Medical Imaging, 16, 852-863.

Troxler, D. (1804). Über das Verschwinden gegebener Gegenstände innerhalb unseres Gesichtskreises. In K. Himly \& J. A. Schmidt (Eds.), Ophthalmische Bibliothek (pp. 51-53). Jena: Frommann.

Wandell, B. A., Chial, S., \& Backus, B. T. (2000). Visualization and measurement of the cortical surface. Journal of Cognitive Neuroscience, 12, 739-752.

Weil, R. S., Kilner, J. M., Haynes, J. D., \& Rees, G. (2007). Neural correlates of perceptual filling-in of an artificial scotoma in humans. Proceedings of the National Academy of Sciences, U.S.A., 104, 5211-5216.

Weil, R. S., Watkins, S., \& Rees, G. (2008). Neural correlates of perceptual completion of an artificial scotoma in human visual cortex measured using functional MRI. Neuroimage, 42, 1518-1528.

Wilke, M., Logothetis, N. K., \& Leopold, D. A. (2006). Local field potential reflects perceptual suppression in monkey visual cortex. Proceedings of the National Academy of Sciences, U.S.A., 103, 17507-17512.

Windmann, S., Wehrmann, M., Calabrese, P., \& Güntürkün, O. (2006). Role of the prefrontal cortex in attentional control over bistable vision. Journal of Cognitive Neuroscience, $18,456-471$. 